\title{
BCAT1 decreases the sensitivity of cancer cells to cisplatin by regulating mTOR-mediated autophagy via branched-chain amino acid metabolism
}

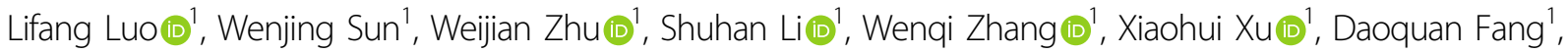 \\ Tan Hooi Min Grahn (1) ${ }^{2}$, Lei Jiang (10) and Yihu Zheng (10 ${ }^{3}$
}

\begin{abstract}
Cisplatin is one of the most effective chemotherapy drugs and is widely used in the treatment of cancer, including hepatocellular carcinoma (HCC) and cervical cancer, but its therapeutic benefit is limited by the development of resistance. Our previous studies demonstrated that BCAT1 promoted cell proliferation and decreased cisplatin sensitivity in HCC cells. However, the exact role and mechanism of how BCAT1 is involved in cisplatin cytotoxicity remain undefined. In this study, we revealed that cisplatin triggered autophagy in cancer cells, with an increase in BCAT1 expression. The cisplatin-induced up-regulation of BCAT1 decreased the cisplatin sensitivity by regulating autophagy through the mTOR signaling pathway. In addition, branched-chain amino acids or leucine treatment inhibited cisplatin- or BCAT1-mediated autophagy and increased cisplatin sensitivity by activating mTOR signaling in cancer cells. Moreover, inhibition of autophagy by chloroquine increased cisplatin sensitivity in vivo. Also, the knockdown of BCAT1 or the administration of leucine activated mTOR signaling, inhibited autophagy, and increased cisplatin sensitivity in cancer cells in vivo. These findings demonstrate a new mechanism, revealing that BCAT1 decreases cisplatin sensitivity in cancer cells by inducing mTOR-mediated autophagy via branched-chain amino acid leucine metabolism, providing an attractive pharmacological target to improve the effectiveness of chemotherapy.
\end{abstract}

\section{Introduction}

Cisplatin, a broad-spectrum anti-tumor chemotherapeutic agent, is used in clinical practice for the treatment of various types of tumors ${ }^{1}$, including hepatocellular carcinoma (HCC) and cervical cancer. However, the development of cisplatin resistance leads to treatment failure $^{2}$. Although significant progress has been made in understanding the mechanisms of cisplatin drug resistance in human cancer, effective strategies to improve cisplatin sensitivity are still lacking. Branched-chain

\footnotetext{
Correspondence: Yihu Zheng (zhengyihu@foxmail.com) or

Lei Jiang (jiangleistone79@163.com)

${ }^{1}$ Central Laboratory, the First Affiliated Hospital of Wenzhou Medical University, Wenzhou 325000, China

2Division of Molecular Medicine and Gene Therapy, Lund Stem Cell Center, Lund University Hospital, Lund 22184, Sweden

Full list of author information is available at the end of the article

Edited by J.-E. Ricci
}

amino acid transaminase 1 (BCAT1) is a cytosolic enzyme that catalyzes the synthesis of $\alpha$-ketoglutarates from branched-chain amino acids (BCAAs) and then to produce glutamates and branched-chain $\alpha$-ketoacids ${ }^{3}$. It is involved in the progression of various cancer, including breast $^{4}$, stomach ${ }^{5}$, esophageal ${ }^{6}$, lung ${ }^{7}$, pancreatic ${ }^{8}$, prostate $^{9}$, cervical $^{10}$ and liver cancer ${ }^{11,12}$. Our previous studies showed that BCAT1 expression correlated with a poor prognosis of HCC patients and promoted tumor cell growth in vitro and in vivo ${ }^{11}$. Moreover, BCAT1 decreased the cisplatin sensitivity of HCC cells ${ }^{11}$. Several studies also show that BCAT1 overexpression is associated with multi-drug resistance ${ }^{3,9,13}$, including bevacizumab on glioblastoma ${ }^{3}$, EGFR tyrosine kinase inhibitors on lung cancer ${ }^{13}$ and cisplatin on prostate cancer ${ }^{9}$. However, the exact role and mechanism of the

\section{(c) The Author(s) 2021}

(c) (i) Open Access This article is licensed under a Creative Commons Attribution 4.0 International License, which permits use, sharing, adaptation, distribution and reproduction in any medium or format, as long as you give appropriate credit to the original author(s) and the source, provide a link to the Creative Commons license, and indicate if changes were made. The images or other third party material in this article are included in the article's Creative Commons license, unless indicated otherwise in a credit line to the material. If material is not included in the article's Creative Commons license and your intended use is not permitted by statutory regulation or exceeds the permitted use, you will need to obtain permission directly from the copyright holder. To view a copy of this license, visit http://creativecommons.org/licenses/by/4.0/. 
involvement of BCAT1 in cisplatin cytotoxicity remain undefined.

BCAT1 is an enzyme responsible for initiating the catabolism of BCAAs, such as leucine (Leu), thus providing macromolecule precursors ${ }^{14}$. BCAAs are well-known activators of mammalian target of rapamycin $(\mathrm{mTOR})^{15}$, especially Leu ${ }^{16}$. Moreover, the activation of mTOR inhibits autophagy flux ${ }^{17}$. Autophagy is an important homeostasis program that is responsible for removing dysfunctional or damaged organelles in all living cells ${ }^{18}$. Various stimulations, such as starvation, growth factor withdrawal, hypoxia and genotoxic or oxidative stress, can induce autophagy ${ }^{19,20}$. In cancer cells, autophagy is considered a double-edged sword, because it has tumorpromoting and tumor-suppressing properties ${ }^{18}$. Currently, the most popular view is that autophagy kills cancer cells during the tumorigenesis stage, inhibits tumor growth, and after tumorigenesis, autophagy protects cancer cell survival against various harsh environments ${ }^{20}$. Autophagy is suggested to mediate a cancer cell's resistance to chemotherapy and radiation treatment ${ }^{21,22}$. Moreover, various studies show that autophagy contributes to cisplatin chemoresistance, and inhibiting autophagy, perhaps, is a means for overcoming cisplatin resistance $^{23-25}$.

In this study, we demonstrated a new mechanism of cisplatin resistance mediated by BCAT1. Our results showed that the cisplatin-induced the upregulation of BCAT1, which decreased the cisplatin sensitivity of cancer cells by facilitating autophagy via the Leu-mediated activation of the mTOR signaling pathway.

\section{Results}

\section{BCAT1 expression decreases cisplatin cytotoxicity in} cancer cells

The expression levels of BCAT1 were detected by qRTPCR and Western blotting in the cervical cancer cell line (Hela) and HCC cell lines (HepG2 and Huh-7) treated with cisplatin. The mRNA and protein expression levels of BCAT1 were significantly increased in the Hela, HepG2, and Huh-7 cells after the cisplatin treatment (Fig. 1A-B and S1A). The protein expression levels of p53 were significantly increased in Hela, HepG2, and Huh-7 cells after the cisplatin treatment (Fig. 1B and S1A). Furthermore, the BCAT1 protein level in the HepG2 cells was higher than in the Hela cells (Fig. 1C) and the HepG2 cells with a high expression of BCAT1 were less sensitive to cisplatin compared with the Hela cells with a low expression of BCAT1 (Fig. 1D). To determine the functional role of BCAT1 with respect to cisplatin sensitivity, BCAT1 was overexpressed in the Hela and Huh-7 cells, which express low levels of BCAT1 (Fig. 1C and S1B), using a lentiviral vector. In addition, BCAT1 expression was knocked down in the HepG2, which express a high level of BCAT1, using shRNA. The increased BCAT1 protein level in the Hela cells (Fig. 1E) and Huh-7 cells (Figure S1C) transfected with the lenti-BCAT1 was verified by Western blotting. The overexpression of BCAT1 significantly decreased the sensitivity of the Hela (Fig. 1F) and Huh-7 (Figure S1D) cells to cisplatin. Conversely, the knockdown of BCAT1 by shRNA greatly enhanced the cytotoxicity of cisplatin in the HepG2 cells (Fig. 1G-H).

\section{Cisplatin-induced autophagy decreases cisplatin cytotoxicity}

We assessed the expression level of p62 and the conversion of the LC3B-I into LC3B-II after treating the Hela, HepG2, and Huh-7 cells with cisplatin. The Western blot results showed an increase in the lipidated form of LC3 (LC3B-II) and a reduction in p62 expression after cisplatin treatment in Hela, HepG2, and Huh-7 cell lines (Fig. 2A, $\mathrm{B}$ and S2A-B). Although the changes in LC3B-II and p62 levels indicate the autophagic flux, the fluorescentlytagged pCMV-Cherry-GFP-LC3B vector can be used to monitor the autophagic flux more reliably ${ }^{26}$. Thus, the Hela or HepG2 cells were transiently transfected for $24 \mathrm{~h}$ with pCMV-Cherry-GFP-LC3B, and then, the cells were treated with cisplatin for $24 \mathrm{~h}$. There was an increase in the mCherry positive signal after the cisplatin treatment (Figure S2C). Treatment with the autophagy inhibitor CQ or 3-MA restored the expression of p62 (Fig. 2A-D and S2A-B). Inhibition of autophagy at late stage by CQ increased the level of LC3B-II whereas suppression of autophagy initiation by 3-MA decreased the level of LC3BII (Fig. 2A-D and S2A-B). To determine whether cisplatininduced autophagy alters the sensitivity, Hela, HepG2, and Huh-7 cells were treated with increasing concentrations of cisplatin alone or combined with CQ or 3-MA. Interestingly, the inhibition of autophagy significantly enhanced the sensitivity of cells to cisplatin (Fig. 2E-H and S2D-E). Apoptosis and cell cycle analysis also showed that cisplatin treatment induced cell apoptosis (Figure S3A) and reduced number of cells in G2/M phase (Figure S3B). CQ or 3-MA significantly increased cisplatin-induced apoptosis (Figure S3A) and also reduced the proportion of cells in G2/M phase after cisplatin treatment (Figure S3B). These data indicated that autophagy does play a specific role in regulating cisplatin sensitivity.

\section{BCAT1 decreases cisplatin sensitivity by activating autophagy via the mTOR pathway}

As shown in Fig. 3A and S4A, the overexpression of BCAT1 increases LC3B-II expression and suppresses p62 expression in Hela and Huh-7 cells, whereas the knockdown of BCAT1 decreases LC3B-II expression and increases p62 expression in HepG2 cells (Fig. 3B), indicating that BCAT1 induces autophagy in cervical cancer 

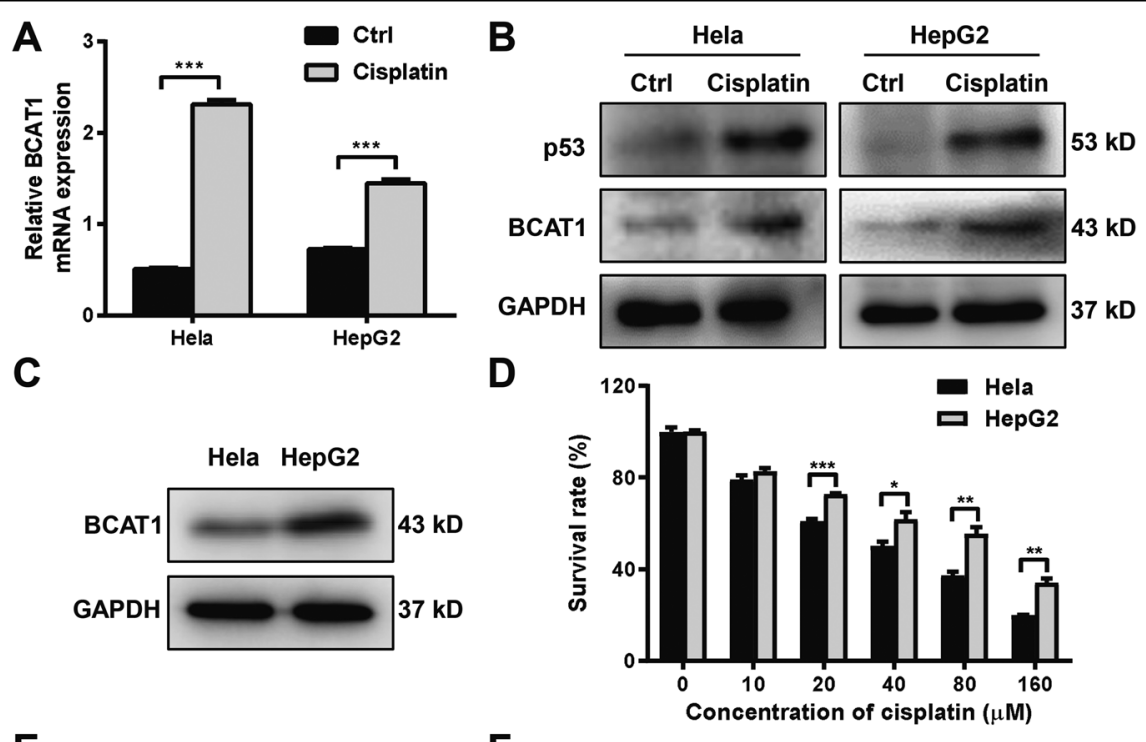

E
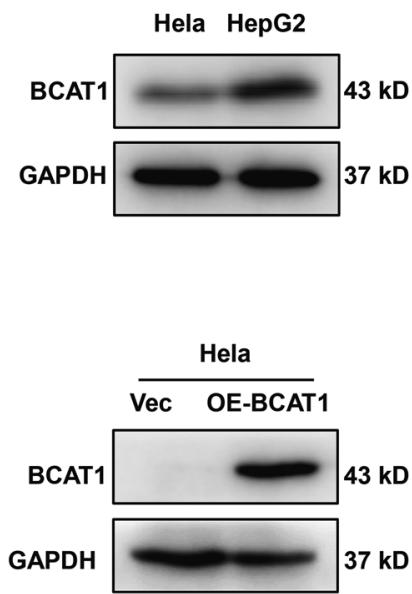

G

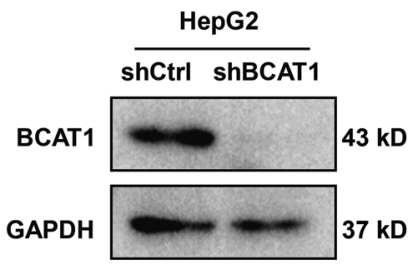

$\mathbf{F}$

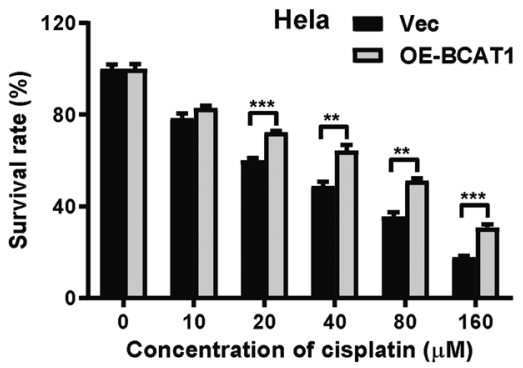

H

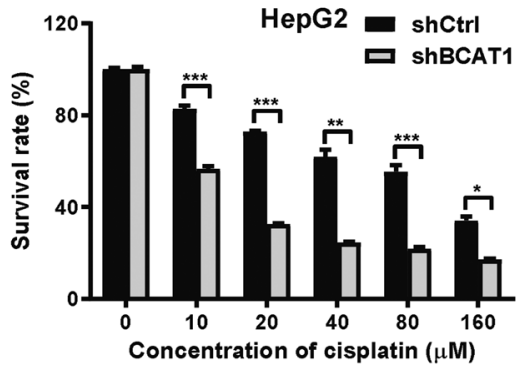

Fig. 1 BCAT1 expression decreases cisplatin sensitivity. A qRT-PCR was performed to detect BCAT1 mRNA expression in Hela and HepG2 cells treated with cisplatin. Hela cells were treated with $20 \mu \mathrm{M}$ cisplatin and HepG2 cells were treated with $10 \mu \mathrm{M}$ cisplatin for $24 \mathrm{~h}$. B Western blot analysis of BCAT1 and p53 protein expression in Hela and HepG2 cells treated with cisplatin ( 20 and $10 \mu \mathrm{M}$, respectively) for $24 \mathrm{~h}$. C Western blot analysis of BCAT1 protein expression in Hela and HepG2 cell lines. D CCK-8 assays were performed to measure the survival rate of Hela and HepG2 cells treated with different concentrations of cisplatin for $24 \mathrm{~h}$. E A Western blot was used to verify the increased protein level of BCAT1 in Hela cells overexpressing BCAT1. F CCK-8 assays were performed to examine the cytotoxicity in Hela cells overexpressing BCAT1 that were treated with different concentrations of cisplatin for $24 \mathrm{~h}$. G A Western blot was used to verify the decreased protein level of BCAT1 in the BCAT1 knockdown HepG2 cells. H CCK-8 assays were used to examine the cytotoxicity in the BCAT1 knockdown HepG2 cells treated with different concentrations of cisplatin for $24 \mathrm{~h}$. Vec, empty vector; Ctrl, control. Three independent experiments were performed. ${ }^{*} P<0.05$, ${ }^{* *} P<0.01,{ }^{* * *} P<0.001$.

and HCC cells. To further explore the specific mechanism of BCAT1-regulated autophagy, we used the PEX100 Phospho Explorer Array to identify specific BCAT1 downstream effectors that regulate autophagy. The knockdown of BCAT1 expression significantly increased the phosphorylation of 4E-BP1 and p70S6K in the HepG2 cells (Figure S4B-C), indicating that the inhibition of
BCAT1 expression might activate the mTOR signaling pathway. Consistent with the PEX100 Phospho Explorer Array results, the Western blot data demonstrated that the overexpression of BCAT1 decreased the phosphorylation of 4E-BP1, p70S6K, and mTOR in the Hela cells (Fig. 3C) and Huh-7 cells (Figure S4D), whereas the knockdown of BCAT1 significantly increased the 

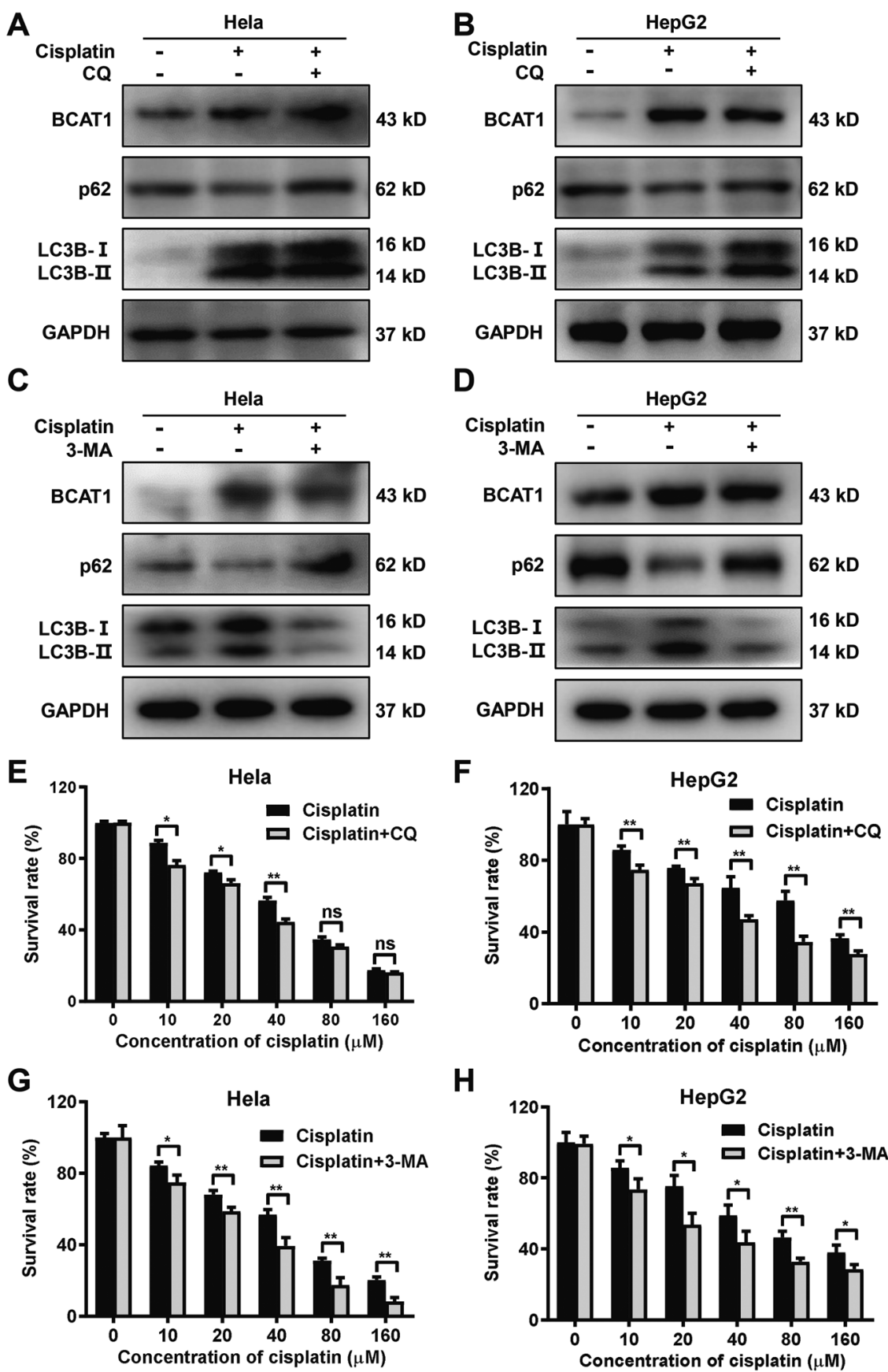

Fig. 2 Cisplatin-induced autophagy confers decreased cisplatin cytotoxicity. A, B Western blot analysis of BCAT1, p62, and LC3-I/II protein levels in the Hela (A) and HepG2 (B) cells treated with cisplatin (20 $\mu \mathrm{M}$ and $10 \mu \mathrm{M}$, respectively) alone or in combination with $20 \mu \mathrm{M}$ chloroquine (CQ) for $24 \mathrm{~h}$. C, D Western blot analysis of BCAT1, p62, and LC3-1/II protein levels in the Hela (C) and HepG2 (D) cells treated with cisplatin $(20 \mu \mathrm{M}$ and $10 \mu \mathrm{M}$, respectively) alone or in combination with $2 \mathrm{mM}$ 3-methyladenine (3-MA) for $24 \mathrm{~h}$. E, F Cell viability was assessed by CCK-8 assays. Hela (E) and HepG2 (F) cells were incubated with increasing concentrations of cisplatin alone or in combination with CQ $(20 \mu \mathrm{M})$ for 24 h. G, H Cell viability was assessed by CCK-8 assays. Hela $(\mathbf{G})$ and HepG2 $(\mathbf{H})$ cells were incubated with increasing concentrations of cisplatin alone or in combination with 3-MA (2 mM) for $24 \mathrm{~h}$. Three independent experiments were performed. ns, not significant. ${ }^{*} P<0.05$, ${ }^{* *} P<0.01$.

phosphorylation of 4E-BP1, p70S6K and mTOR in the HepG2 cells (Fig. 3D). Moreover, the knockdown of BCAT1 restored the cisplatin-induced inhibition of the
mTOR phosphorylation level and reversed cisplatininduced autophagy (Fig. 3E). Next, the HepG2 cells were transiently transfected with pCMV-Cherry-GFP-LC3B for 


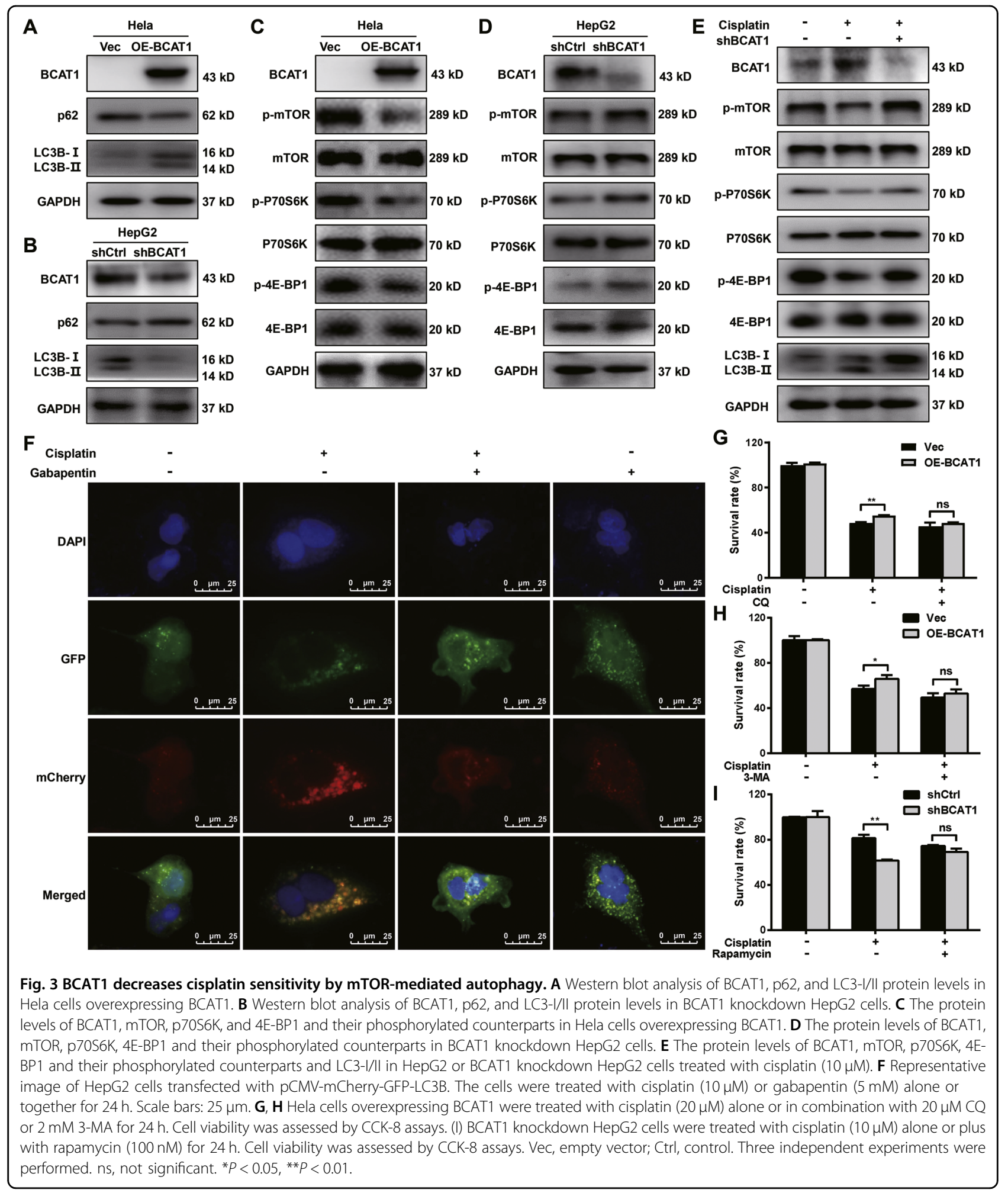

$24 \mathrm{~h}$, and then, the cells were treated with cisplatin or the BCAT1 inhibitor gabapentin alone or together for $24 \mathrm{~h}$. The results revealed that the increased mCherry positive signals induced by the cisplatin treatment were reversed by inhibiting BCAT1 with gabapentin in the HepG2 cells (Fig. 3F).

To determine the role of BCAT1-triggered autophagy in cisplatin cytotoxicity, we used autophagy inhibitors or 
activators to treat the Hela and Huh-7 cells with BCAT1 overexpression or the HepG2 cells with BCAT knockdown, respectively. The overexpression of BCAT1 decreased the sensitivity of Hela (Fig. 3G-H) and Huh-7 (Figure S4E-F) cells to cisplatin, and the autophagy inhibitors CQ and 3-MA reversed this effect (Fig. 3G-H and Figure S4E-F). In contrast, the mTOR inhibitor rapamycin blocked the increased cisplatin sensitivity in the HepG2 cells that was induced by the knockdown of BCAT1 (Fig. 3I). As shown in Figure S5A-C, overexpression of BCAT1 decreased cisplatin-induced apoptosis in Hela and Huh-7 cells, whereas knockdown of BCAT1 increased cisplatininduced apoptosis in HepG2 cells. CQ treatment promoted cisplatin-induced apoptosis and reversed the effect of BCAT1 overexpression (Figure S5A-B). In contrast, rapamycin treatment suppressed cisplatin-induced apoptosis (Figure S5C). These results suggested that BCAT1 decreased the cisplatin sensitivity by regulating autophagy and by affecting the mTOR signaling pathway.

BCAAs and leucine decrease cisplatin-mediated autophagy and increase cisplatin sensitivity

To investigate the role of BCAAs or Leu in cisplatin sensitivity, we treated the Hela and Huh-7 cells with BCAAs or Leu combined with cisplatin. The BCAAs or Leu treatment reversed the decreased mTOR, 4E-BP, and p70S6K phosphorylation and the increased cisplatininduced autophagy in the Hela (Fig. 4A, B) and Huh-7 (Figure S6A-B) cells. Moreover, the BCAAs or Leu treatment significantly increased the sensitivity of the Hela (Fig. 4C, D) and Huh-7 (Figure S6C-D) cells to cisplatin. Apoptosis and cell cycle analysis also showed that BCAAs or Leu treatment significantly increased cisplatininduced apoptosis (Figure S3A) and reduced the proportion of cells in $\mathrm{G} 2 / \mathrm{M}$ phase after cisplatin treatment (Figure S3B). The Hela cells were transiently transfected for $24 \mathrm{~h}$ with pCMV-Cherry-GFP-LC3B, and then, the cells were treated with cisplatin or Leu alone or together for $24 \mathrm{~h}$. The results revealed that the increased mCherry positive signals induced by the cisplatin treatment were reversed by the addition of Leu (Fig. 4E). The above experiments indicated that BACC Leu participates in the sensitivity of the cancer cells to cisplatin.

\section{BCAT1 enhances autophagy and inhibits cisplatin sensitivity in cancer cells via leucine reduction}

The Western blot results demonstrated that the Leu treatment reversed the decreased mTOR, 4E-BP and p70S6K phosphorylation levels and the increased autophagy induced by BCAT1 overexpression in the Hela (Fig. 5A) and Huh-7 (Figure S7A) cells. In comparison, the Leu antagonist, Ac-Leu-NH2, reversed the increased mTOR, 4E-BP and p70S6K phosphorylation levels and the decreased autophagy induced by BCAT1 knockdown in the HepG2 cells (Fig. 5B). Moreover, the addition of Leu blocked the decreased cisplatin cytotoxicity in the Hela (Fig. 5C) and Huh-7 (Figure S7B) cells that was induced by the overexpression of BCAT1. In contrast, the increased cisplatin sensitivity caused by BCAT1 knockdown was reversed by Ac-Leu-NH2 in the HepG2 cells (Fig. 5D). As shown in the Figure S5A-C, the treatment of Leu significantly increased the apoptotic ratio in Hela and Huh-7 cells with BCAT1 overexpression, while the effect of Ac-Leu-NH2 was just opposite in HepG2 cells with BCAT1 knockdown.

\section{Knockdown of BCAT1 increases cisplatin sensitivity in vivo}

We further explored the role of BCAT1 in cisplatin sensitivity in vivo. As shown in Fig. 6A, B, individually, the knockdown of BCAT1 or cisplatin therapy in the HepG2 cells reduced the tumor volume. However, tumors from the cells with BCAT1 knockdown exhibited a significantly enhanced response to cisplatin, leading to a significant reduction in tumor size (Fig. 6A, B) and weight (Fig. 6C). During the treatment, no obvious change in the bodyweight of the mice was found (Fig. 6D). Moreover, the Western blot results confirmed that the knockdown of BCAT1 reversed the cisplatin-induced decrease in the mTOR phosphorylation level and increase in autophagy in tumor tissues (Fig. 6E). Immunohistochemistry analysis also showed that the knockdown of BCAT1 increased the cisplatin sensitivity, resulting in higher expression levels of cleaved caspase- 3 and TUNEL, and a lower expression level of Ki-67 in tumor tissues (Fig. 6F).

\section{Inhibition of autophagy or the administration of Leu increases the cisplatin sensitivity of cancer cells in vivo}

Next, we investigated whether Leu or autophagy was involved in the cisplatin sensitivity of cancer cells in vivo. We used the autophagy inhibitor CQ or Leu combined with the cisplatin treatment in tumor-harboring mice. The Hela cells were subcutaneously injected into the flanks of the nude mice. The tumor-harboring mice were randomly divided into 6 groups as follows: Control, Cisplatin, CQ, Leu, Cisplatin + CQ, and Cisplatin + Leu. The tumor growth curves showed that the CQ or Leu treatment significantly enhanced the response of the tumor cells to cisplatin in vivo, which resulted in a significant reduction in tumor size (Fig. 7A-C) and weight (Fig. 7D, E). However, no significant change in the bodyweight of the mice was observed (Fig. 7F, G). In addition, in accordance with the in vitro results, the Western blot data revealed that the Leu treatment reversed the cisplatininduced decrease in the mTOR phosphorylation level and increase in autophagy (Fig. 7H). IHC analysis showed that the treatment of CQ or Leu significantly increased the cytotoxicity of the cisplatin, resulting in higher expression levels of cleaved caspase- 3 and TUNEL, and a lower 

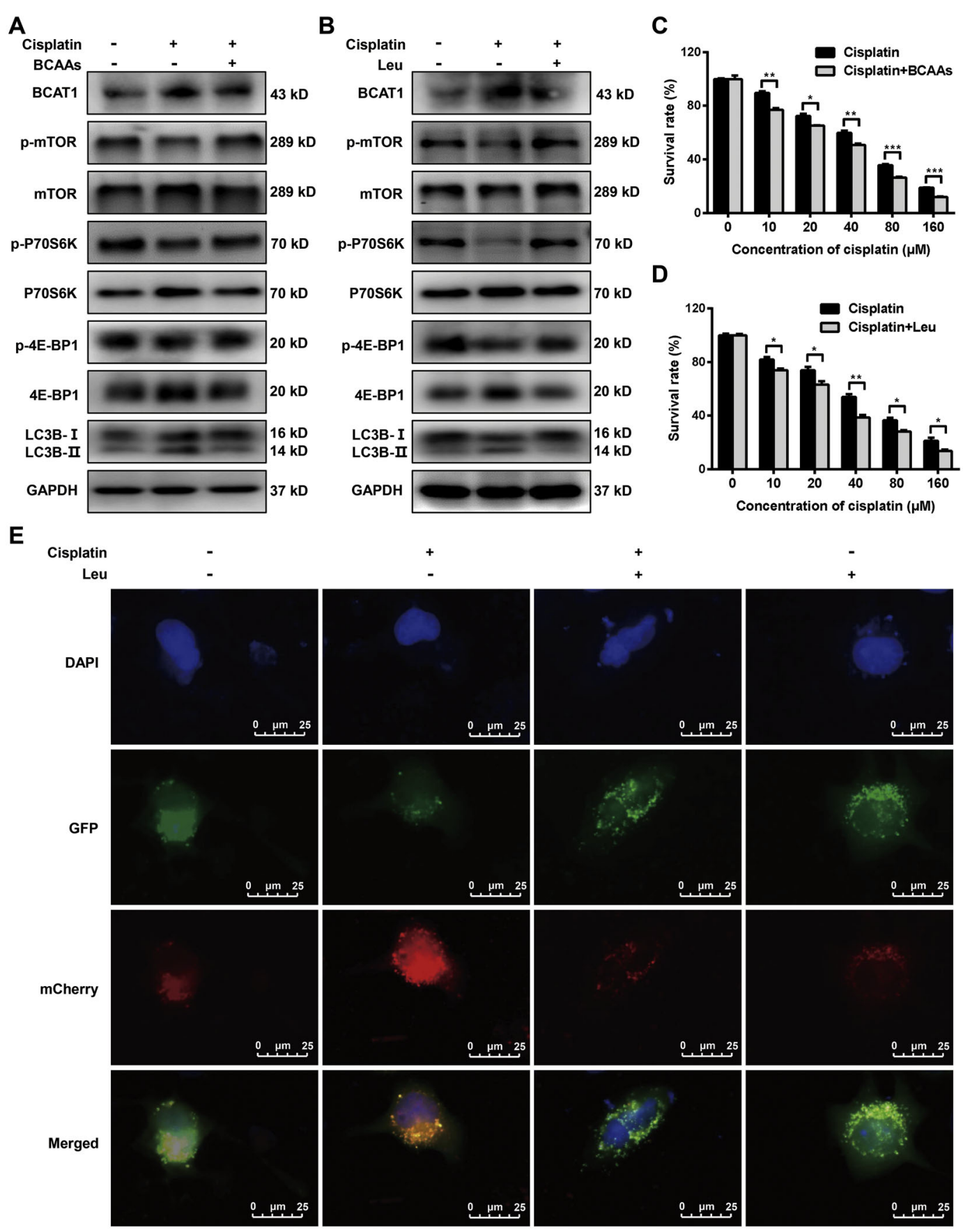

Fig. 4 Branched-chain amino acids (BCAAs) and leucine (Leu) decrease cisplatin-mediated autophagy and increase cisplatin sensitivity. A B Western blot analysis of the protein levels of BCAT1, mTOR, p70S6K, 4E-BP1 and their phosphorylated counterparts and LC3-I/II in Hela cells treated with cisplatin $(20 \mu \mathrm{M})$ or in combination with BCAAs $(5 \mathrm{mM})$ or Leu $(2 \mathrm{mM})$ for $24 \mathrm{~h}$. C, D Hela cells were treated with increasing concentrations of cisplatin alone or in combination with BCAAs $(5 \mathrm{mM})$ or Leu $(2 \mathrm{mM})$ for $24 \mathrm{~h}$. Cell viability was assessed by CCK-8 assays. E Representative image of Hela cells transfected with pCMV-mCherry-GFP-LC3B. The cells were treated with cisplatin $(20 \mu \mathrm{M})$ or Leu $(2 \mathrm{mM})$ alone or together for $24 \mathrm{~h}$. Scale bars: $25 \mu \mathrm{m}$. Three independent experiments were performed. ${ }^{*} P<0.05,{ }^{* *} P<0.01,{ }^{* *} P<0.001$.

expression level of Ki-67 (Fig. 7I). These data suggest that the Leu treatment or inhibition of autophagy by CQ indeed altered the sensitivity of the cancer cells to cisplatin in vivo. It is worth noting that BCAT1/Leu/ mTOR/Autophagy is a concise mechanism for the cisplatin sensitivity of cervical cancer and HCC cells (Fig. 7J). 

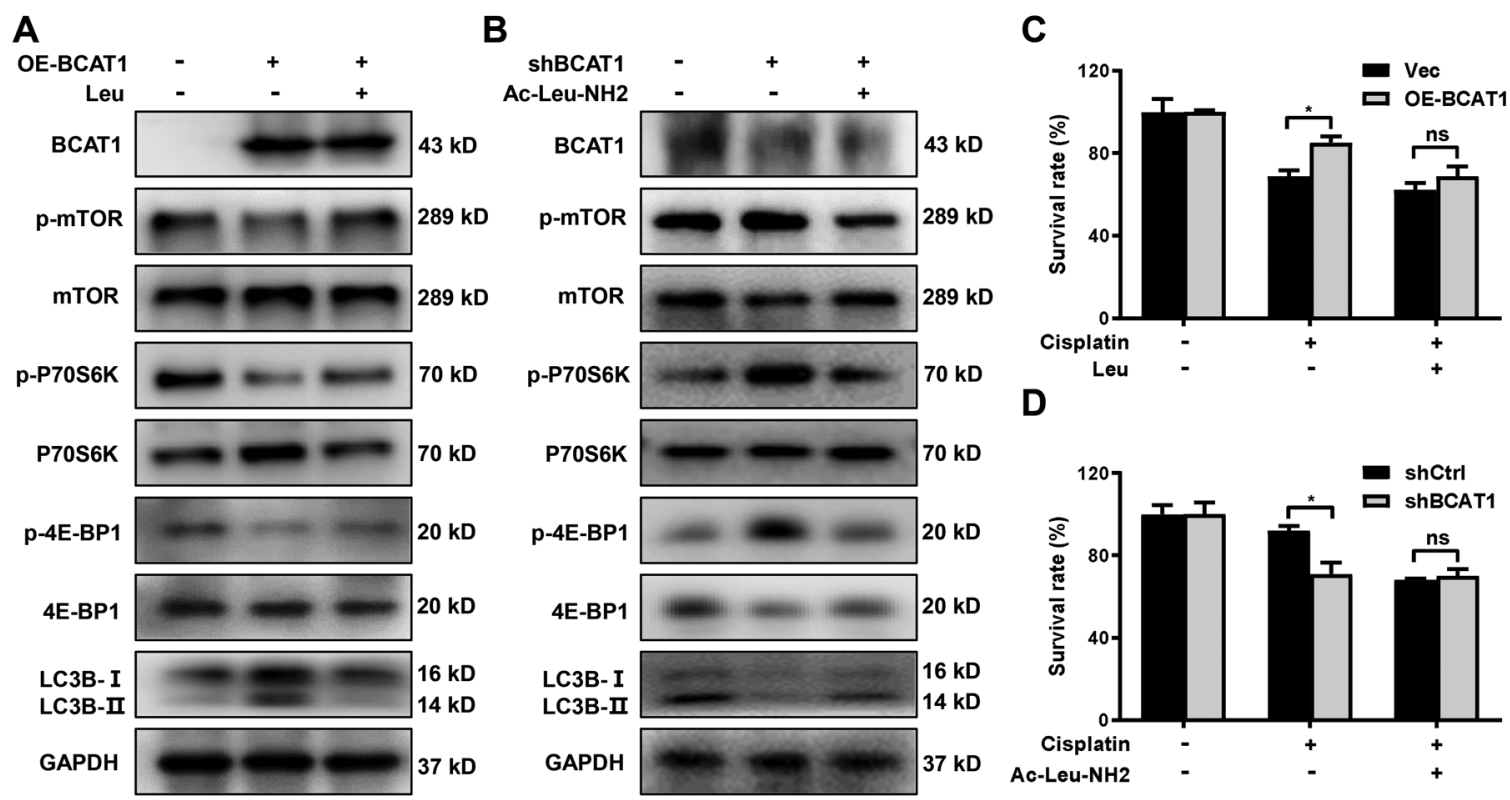

Fig. 5 BCAT1 enhances autophagy and decreases cisplatin sensitivity via leucine (Leu) reduction. A Western blot analysis of the protein levels of BCAT1, mTOR, p70S6K, 4E-BP1 and their phosphorylated counterparts and LC3-I/II in Hela cells with BCAT1 overexpression or in combination with Leu $(2 \mathrm{mM})$ treatment for $24 \mathrm{~h}$. B Western blot analysis of the protein levels of BCAT1, mTOR, p70S6K, 4E-BP1 and their phosphorylated counterparts and LC3-1/II in HepG2 cells with BCAT1 knockdown or in combination with an Ac-Leu-NH2 $(5 \mathrm{mM})$ treatment for $24 \mathrm{~h}$. C Hela cells overexpressing BCAT1 were treated with cisplatin $(20 \mu \mathrm{M})$ alone or in combination with Leu $(2 \mathrm{mM})$ for $24 \mathrm{~h}$. Cell viability was assessed by CCK-8 assays. D BCAT1 knockdown HepG2 cells were treated with cisplatin $(10 \mu \mathrm{M})$ alone or in combination with Ac-Leu-NH2 $(5 \mathrm{mM})$ for $24 \mathrm{~h}$. Cell viability was assessed by CCK-8 assays. Vec, empty vector; Ctrl, control. Three independent experiments were performed. ns, not significant. ${ }^{*} P<0.05$.

\section{Discussion}

BCAT1 expression is confirmed to be associated with cancer progression in various solid tumors ${ }^{4-9,27}$, including cervical cancer ${ }^{10}$ and $\mathrm{HCC}^{12}$. Increased BCAT1 expression correlates with the chemotherapeutic agent resistance ${ }^{3,9,13}$. For instance, Cho et al. ${ }^{3}$ reported that BCAT1 promoted IDH1 wild-type glioblastoma multiforme cell proliferation, invasion and increased resistance to bevacizumab treatment. In addition, Zhu et al. ${ }^{9}$ found that miR-218 increased chemosensitivity to cisplatin by targeting BCAT1 in prostate cancer. Moreover, Wang et al. ${ }^{13}$ showed that BCAT1 conferred sublethal tyrosine kinase inhibitor resistance in EGFR-mutated lung cancer cells through the H3K9 demethylation-mediated BCAA metabolism reprogramming. The above findings confirm the important role of BCAT1 in tumor drug resistance. In our previous study, we demonstrated that the exogenous expression of BCAT1 significantly promoted cell proliferation and decreased cisplatin sensitivity and increased the lipidated form of LC3 (LC3B-II) in HCC cells ${ }^{11}$, indicating that BCAT1 expression and autophagy have been implicated in cisplatin sensitivity. However, the molecular mechanism of how BCAT1 regulates cisplatin chemosensitivity in cancer cells remains unclear. In this study, we further revealed that BCAT1-regulated cisplatin sensitivity in cervical cancer (Hela) and HCC (HepG2 and
Huh-7) cells in vitro and in vivo. Furthermore, we demonstrated a new mechanism that the cisplatininduced up-regulation of BCAT1 decreased the cisplatin sensitivity in cervical cancer and HCC cells by regulating mTOR-mediated autophagy via BCAA metabolism.

Previous studies confirmed that autophagy participates in tumor drug resistance. For example, Pennati et al. ${ }^{23}$ found that autophagy decreased cisplatin cytotoxicity in castration-resistant prostate cancer cells. In addition, $\mathrm{Yu}$ et al. ${ }^{24}$ showed that the induction of autophagy counteracts the anticancer effect of cisplatin in acquired drugresistant human esophageal cancer cells. Moreover, Zhao et $\mathrm{al}^{25}$ demonstrated that the inhibition of autophagy sensitized gastric cancer cells to cisplatin. In this study, we revealed that cisplatin triggered autophagy in cancer cells with an increase in BCAT1 expression. We further confirmed that BCAT1 overexpression induced autophagy and decreased cisplatin sensitivity, whereas the knockdown of BCAT1 inhibited autophagy and increased cisplatin sensitivity. CQ is a classic autophagy lysosomal inhibitor. In our study, the CQ and 3-MA treatment significantly enhanced the sensitivity of the cervical cancer and HCC cells to cisplatin in vivo and in vitro and partially relieved the BCAT1-induced resistance to cisplatin. These data indicated that the cisplatin-induced upregulation of BCAT1 reduced the sensitivity of the 


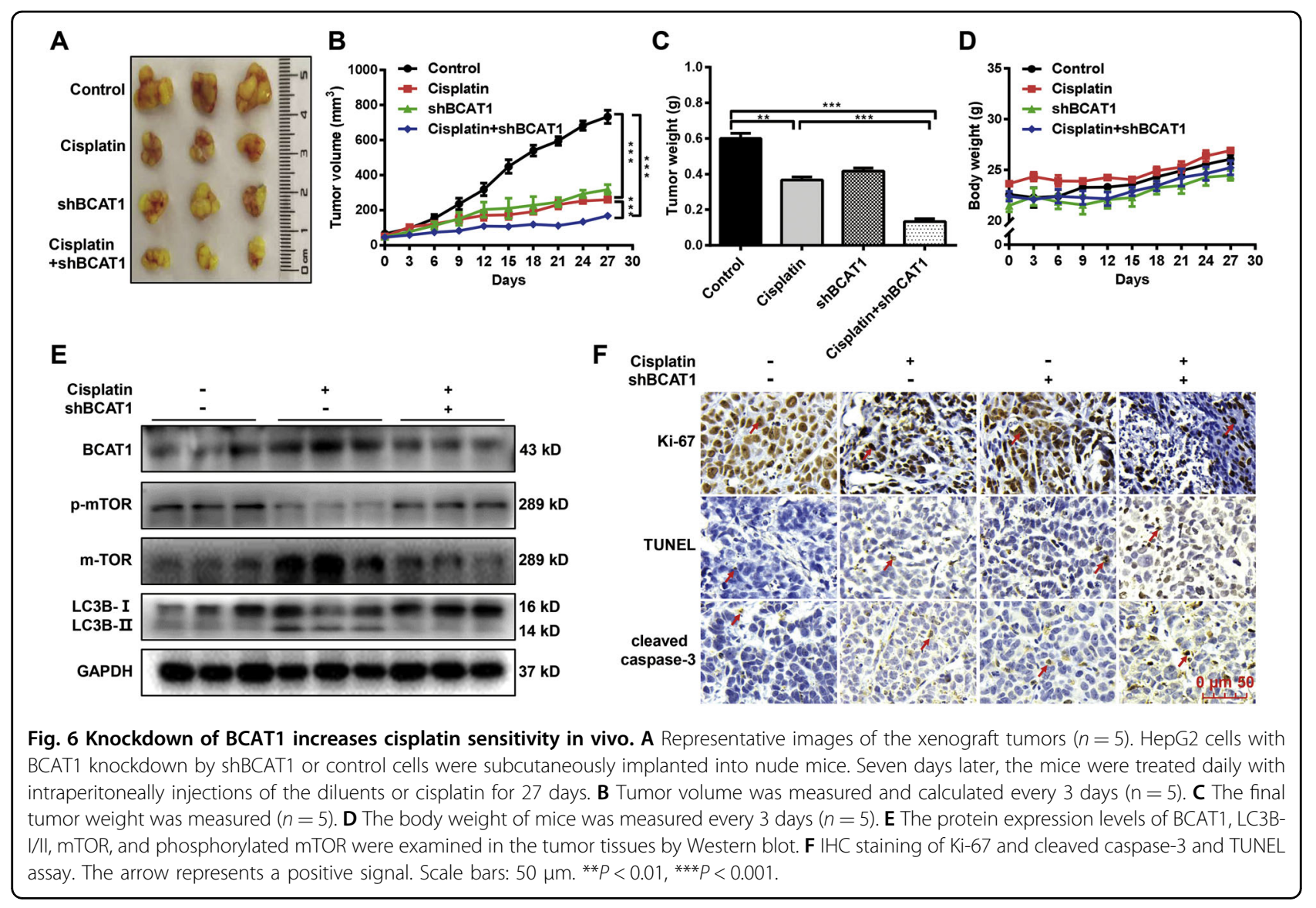

cervical cancer and HCC cells to cisplatin through an autophagy-related mechanism.

In order to explore the potential regulatory mechanism of BCAT1-induced autophagy, we utilized a Phospho Explorer Array to predict the potential downstream targets. The results showed that the knockdown of BCAT1 expression significantly increased the phosphorylation of 4E-BP1 and p70S6K, which are important downstream effectors of the mTOR signaling pathway ${ }^{28}$, suggesting that the inhibition of BCAT1 expression activates the mTOR signaling pathway. The Western blot data also demonstrated that BCAT1 overexpression suppressed the mTOR signaling pathway (by decreasing the phosphorylation of mTOR, 4E-BP1, and p70S6K). Whereas BCAT1 knockdown activated the mTOR signaling pathway (by increasing the phosphorylation of mTOR, 4E-BP1, and p70S6K). Considering that the serine/threonine kinase, mTOR pathway is the classical pathway that regulates autophagy $^{29}$, we speculated that BCAT1 affected the cisplatin sensitivity of cancer cells by regulating autophagy through the mTOR pathway. Specifically, rapamycin, an inhibitor of the mTOR signaling pathway, decreased cisplatin sensitivity in the BCAT1 knockdown HepG2 cells.

BCAT1 is the key enzyme responsible for the transformation of BCAAs in vivo ${ }^{30,31}$, and BCAAs are described as a nutrient signal that activates the mTOR signaling pathway $^{32}$. In addition, the potential beneficial effects of BCAAs have also been reported ${ }^{33,34}$. BCAAs administration shows a long-term inhibitory effect on carcinogenesis and improves event-free survival and overall survival ${ }^{33}$. Early partial replacement with BCAA-enriched nutrients in the patient's diet may consequently improve the treatment outcome of HCC patients ${ }^{34}$. Therefore, we tried to explore whether BCAAs were involved in BCAT1mediated cisplatin resistance through autophagy. Our results revealed that the BCAAs treatment reversed the cisplatin-induced mTOR signal inhibition and autophagy and restored the sensitivity of the cancer cells to cisplatin. As the most bioactive BCAA, Leu alone has the ability to activate mTOR pathway ${ }^{35}$. We found that Leu treatment also promoted mTOR activation, reduced autophagy flux, and increased the cisplatin cytotoxicity to cancer cells. Moreover, treatment with Leu reversed the mTOR signal inhibition and autophagy induced by BCAT1 overexpression, whereas Leu antagonist (Ac-Leu-NH2) reversed the mTOR activation and autophagy reduction caused by BCAT1 knockdown. These findings provided compelling evidence that BCAT1-induced autophagy and decreased the sensitivity of the cancer cells to cisplatin via a Leu-regulated mTOR signaling pathway. 


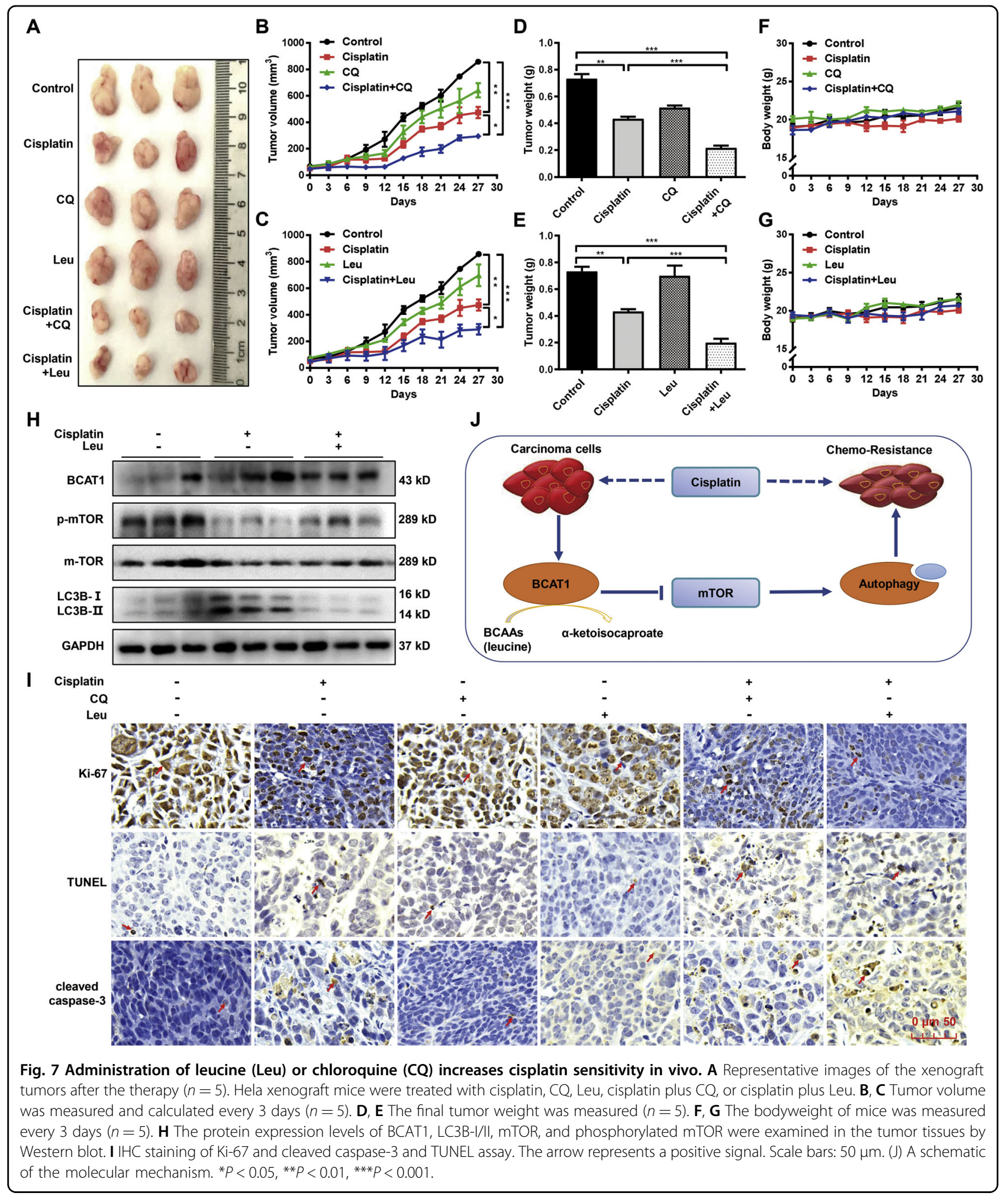

However, there are several limitations of our study. (1) Further studies with the cisplatin-resistant cell lines and patient samples are needed to corroborate our conclusion; (2) In our study, tumor cell lines were directly transplanted into immunocompromised mice to form Cell line Derived Xenograft (CDX) models. However, CDX models have been shown to be limited predictors of clinical outcome ${ }^{36}$. The complexity of tumor 
microenvironment, host immune responses, and tumor heterogeneity pose challenges to clinical success. Alternatively, tumors harvested from Genetically Engineered Mice (GEM) can be transplanted and expanded into fully immunocompetent syngeneic hosts, forming GEMDerived Allograft (GDA) models ${ }^{36}$. The models can be used for the preclinical studies of chemotherapeutic and small-molecule drugs, but also for the preclinical studies of various immunotherapy drugs. (3) In our study, we found that cisplatin treatment can up-regulate the expression of BCAT1, and BCAT1 plays an important role in cisplatin sensitivity. However, whether this phenomenon exists in other DNA damaging agents or chemotherapeutic drugs remains to be investigated.

In conclusion, we discovered the key role of cisplatininduced BCAT1 in the regulation of autophagy. Our results confirmed that BCAT1 enhances the mTORmediated autophagy via BACC Leu metabolism in cancer cells, which finally leads to a reduced cisplatin sensitivity. These findings strongly suggest that BCAT1/Leu/mTOR/ autophagy signaling represents a novel pathway regulating cisplatin sensitivity, thus providing an attractive pharmacological target for chemotherapy to treat cancer.

\section{Materials and methods Cell lines}

Human cervical cancer cell line (Hela), HCC cell lines (Huh-7 and HepG2), and human Embryonic Kidney $293 \mathrm{~T}$ cell line (HEK293T) were obtained from the Chinese Academy of Sciences (Shanghai, China). All cells were cultured in DMEM (Gibco, Grand Island, NY, USA) supplemented with $10 \%$ fetal bovine serum (SigmaAldrich, St. Louis, MO, USA) and $1 \%$ antibioticantimycotic (Sigma-Aldrich). The cells were incubated in a humidified atmosphere at $37^{\circ} \mathrm{C}$ with $5 \% \mathrm{CO}_{2}$.

\section{Reagents}

The following small molecules were used: cisplatin and Leu (Sigma-Aldrich), chloroquine (CQ), rapamycin, gabapentin, and 3-methyladenine (3-MA) (MedChemExpress, Princeton, NJ, USA), Ac-Leu-NH2 (BACHEM, Switzerland), BCAAs (Anhui BBCA Pharmaceuticals Co., Ltd., China). The cisplatin, CQ, gabapentin, 3-MA, Leu, Ac-Leu-NH2, and BCAAs were dissolved in phosphate buffer saline, and rapamycin was dissolved in dimethyl sulfoxide (Sigma-Aldrich).

\section{Lentiviral vectors and transduction}

Lentiviral vectors expressing the BCAT1 gene or a short-hairpin RNA (shRNA) targeting BCAT1 (shBCAT1) were constructed as previously described ${ }^{11}$. The lentiviral particles were produced as described previously ${ }^{11}$. The cells were seeded into 24-well plates at a density of $2 \times 10^{4} /$ well and were transduced with the lentivirus.

\section{Western blot analysis}

The cells or tissue was lysed in RIPA buffer (Thermo Fisher Scientific, Waltham, MA, USA) and protein concentration was measured using the Pierce BCA Protein Assay Kit (Thermo Fisher Scientific). The total protein $(40 \mu \mathrm{g})$ was electrophoresed using 12\% SDS-PAGE and was then transferred onto polyvinylidene difluoride membranes (Millipore, Bedford, MA, USA). The membranes were blocked in 5\% skim milk (BioFroxx, Germany) and were incubated at $4{ }^{\circ} \mathrm{C}$ with primary antibodies overnight. Subsequently, the membranes were incubated with horseradish peroxidase-conjugated secondary antibody at room temperature for $1 \mathrm{~h}$. The samples were visualized using an enhanced chemiluminescence detection and were analyzed using IMAGE LAB software (Bio-Rad). The following primary antibodies were used: anti-GAPDH (Cat. \# 5174), anti-LC3B (Cat. \# 3868), anti-SQSTM1/p62 (Cat. \# 39749), anti-mTOR (Cat. \# 2983 S), anti-phosphorylated mTOR (Ser2448) (Cat. \# 5536 S), anti-4E-BP1 (Cat. \# 9644 T), antiphosphorylated 4E-BP1 (Thr70) (Cat. \# 9455), anti-p70 S6 Kinase (Cat. \# $2708 \mathrm{~T}$ ) and anti-phosphorylated p70 S6 Kinase (Thr421/Ser424) (Cat. \# 9204) (all were purchased from Cell Signaling Technology, Danvers, MA, USA), anti-p53 was purchased from Abcam (Cat. \# ab 131442, Cambridge, MA, USA), and anti-BCAT1 was purchased from BD Biosciences (Cat. \# 611271, San Jose, CA, USA). The HRP-conjugated secondary antibodies were from Thermo Fisher Scientific (Cat. \# 31460, Cat. \# 31430, Waltham, MA, USA). Three independent experiments were performed.

\section{Real-time quantitative PCR (qPCR)}

The total RNA was extracted from the cells using TRIzol (Thermo Fisher Scientific), and the complementary DNA was synthesized using the PrimeScript reverse transcription reagent Kit (Takara, Japan) according to the manufacturer's protocol. The real-time quantitative PCR (qPCR) was performed using TB Green Premix Ex Taq (Takara) on the ABI QuantStudio 5 realtime PCR system (Applied Biosystems, Warrington, U.K.). The relative quantitation was expressed by using the $\mathrm{Ct}$ values, which were determined from triplicate reactions for each target gene and glyceraldehyde-3-phosphate dehydrogenase (GAPDH). The triplicate $\mathrm{Ct}$ values were averaged, and the GAPDH Ct was subtracted to obtain the $\Delta \mathrm{Ct}$. The $2^{-\Delta \Delta \mathrm{Ct}}$ method was utilized to calculate the gene expression values. The following forward and reverse primers were used, respectively: BCAT1: 5'- CAA CTA TGG AGA ATG GTC CTA $-3^{\prime}$ and $5^{\prime}-$ TGT CCA GTC GCT CTC TTC TCT - $3^{\prime}$; and GAPDH: $5^{\prime}$ - CCA GCC GAG CCA CAT CGC TC $-3^{\prime}$ and $5^{\prime}$ - ATG AGC CCC AGC CTT CTC CAT $-3^{\prime}$. Three independent experiments were performed. 


\section{Cell viability assay}

The cell counting kit-8 (CCK-8) assay was used to assess the cell viability. According to the manufacturer's instructions, the cells (2500 per well) were seeded into 96-well plates in a volume of $100 \mu \mathrm{L}$ of complete medium. After $24 \mathrm{~h}$, the treatment regimen of the various drug combinations was added. Then, after $24 \mathrm{~h}$ of incubation, $10 \mu \mathrm{L}$ of the CCK8 (Dojindo, Japan) solution was added to each well, and the plate was incubated for $3 \mathrm{~h}$. The absorbance value was measured at a wavelength of $450 \mathrm{~nm}$. For the BCAAs, Leu, or Ac-Leu-NH2 treatment experiments, the cells were serum-starved $12 \mathrm{~h}$ prior to the treatment. Three independent experiments were performed.

\section{Cell apoptosis assay}

Cell apoptosis was determined using Annexin V-FITC/ PI apoptosis kit (Multi Sciences) or Annexin V-APC/7AAD apoptosis kit (BD Pharmingen, San Diego, CA, USA). According to the manufacturer's instructions, cells $\left(5 \times 10^{5}\right)$ and incubated with Annexin V and PI or 7-AAD in the dark for $15 \mathrm{~min}$. Apoptotic cells were detected with a flow cytometer (Beckman Coulter Inc, Miami, FL, USA). Three independent experiments were performed.

\section{Cell cycle assay}

The cell cycle was checked using a 7-AAD nucleic acid dye (BD Pharmingen). Briefly, cells $\left(1 \times 10^{6}\right.$ per well) were seeded in the serum-free media for $24 \mathrm{~h}$ and then treated with reagents for $24 \mathrm{~h}$. After treatment, cells were harvested at a density of $5 \times 10^{5}$ cells $/ \mathrm{mL}$ and fixed overnight at $4{ }^{\circ} \mathrm{C}$ in $70 \%$ ethanol. Later, cells were washed with PBS and incubated with 7-AAD in the dark for $20 \mathrm{~min}$. The DNA content of stained cells were analyzed with a flow cytometer (Beckman Coulter Inc). Three independent experiments were performed.

\section{Detection of autophagic flux}

The plasmid pCMV-mCherry-GFP-LC3B was purchased from Beyotime Biotechnology (Shanghai, China). The cells were transiently transfected with the pCMVmCherry-GFP-LC3B vector for $24 \mathrm{~h}$ using the Lipofectamine 3000 Reagent (Invitrogen) and were then treated with the different drugs for $24 \mathrm{~h}$. Autophagic flux was determined by fluorescence microscopy (Leica, Wetzlar, Germany). For the Leu treatment experiments, the cells were serum starved $12 \mathrm{~h}$ prior to the treatment. Three independent experiments were performed.

\section{Phospho explorer antibody microarray analysis of the phosphoproteins}

A PEX100 Phospho Explorer Array (Full Moon BioSystems Inc., Sunnyvale, CA, USA) was used to explore the BCAT1 downstream effectors. The extent of the protein phosphorylation was measured as a ratio of the "phospho" and "non-phospho" values. For the chip image obtained by scanning, the original data were analyzed with GenePix Pro 6.0 software (Axon Instruments, Foster City, CA, USA).

\section{Immunohistochemistry (IHC) and TUNEL assay}

Paraffin-embedded tumor tissue sections were taken for IHC analysis as described previously ${ }^{11}$. The following primary antibodies were used: anti-Ki-67 was purchased from Abcam, and anti-cleaved caspase-3 was purchased from Saville Biotechnology (Wuhan, China). The secondary antibody was from Saville Biotechnology. Cell apoptosis in tumor tissue sections $(4 \mu \mathrm{m})$ were evaluated with a DAB (SA-HRP) TUNEL Cell Apoptosis Detection Kit (Saville Biotechnology).

\section{Animal model experiments}

All the animal experiments were approved by the Animal Experimental Ethics Committee of Wenzhou Medical University. Male BALB/c nude mice (3-4 weeks old, 16-20 g) were purchased from the Beijing Vital River Laboratory Animal Technology Co., Ltd. All the mice were given one week to adapt to the new environment before further experimentation. A tumor xenograft model was conducted by subcutaneously implanting isogenic HepG2 cells $\left(5 \times 10^{6} / 0.2 \mathrm{ml}\right)$ transduced with lentishControl or lenti-shBCAT1 into athymic nude mice. The mice were randomly divided into four groups (5 mice/group) as follows: Control, Cisplatin, shBCAT1, and Cisplatin + shBCAT1 groups. We also established a tumor xenograft model by subcutaneously implanting $5 \times$ $10^{6} / 0.2 \mathrm{ml}$ Hela cells into athymic nude mice. After 7 days, the mice were randomized into the control or treatment groups (5 mice/group) as follows: Control, Cisplatin, CQ, Leu, Cisplatin + CQ, and Cisplatin + Leu groups. The mice were treated daily with intraperitoneally injections of the diluents or cisplatin $\left(2 \mathrm{mg} \cdot \mathrm{kg}^{-1}\right)$ and/or CQ $\left(20 \mathrm{mg} \mathrm{kg}^{-1}\right)$ or Leu $\left(150 \mathrm{mg} \mathrm{kg}^{-1}\right)$ for 27 days. During the drug treatments, the subcutaneous tumor size of the nude mice was calculated using the formula $(0.5 \times$ length $\times$ width $^{2}$ ) and the bodyweight of mice was checked every three days. After 27 days of treatment, the nude mice were sacrificed, and the tumors were removed, weighed. Three representative xenograft tumors in each group were taken pictures.

\section{Statistical analysis}

All the data are proven by three independent experiments and expressed as the means \pm standard deviation (SD). The statistical analyses were performed using GraphPad Prism 7.0 software (San Diego, CA, USA). No samples or mice were excluded from the analysis as outliers. The variance was similar between the groups that are being statistically compared. Differences between groups were analyzed by using student's t-test or one-way 
ANOVA. No samples or mice were excluded from the analysis as outliers. $P<0.05$ was considered statistically significant. The quantitative analyses of Western blot and TUNEL staining results are provided in Supplementary data (Figure S8-9).

\section{Author details}

'Central Laboratory, the First Affiliated Hospital of Wenzhou Medical University, Wenzhou 325000, China. ${ }^{2}$ Division of Molecular Medicine and Gene Therapy, Lund Stem Cell Center, Lund University Hospital, Lund 22184, Sweden. ${ }^{3}$ Department of General Surgery, the First Affiliated Hospital of Wenzhou Medical University, Wenzhou 325000, China

\section{Author contributions}

Y.Z. and L.J. performed study concept and design; L.L. and W.S. finished the experimental plan and implementation; W. Zhu, S.L., W. Zhang, and T.H.M.G. provided analysis and interpretation for data; D.F. provided technical support and data analysis; X.X. revised carefully the manuscript and participated in the completion of supplementary experiments; L.J. and L.L. performed writing, review, and revision of the article and all figures. All authors read and approved the final paper.

\section{Ethics}

All the animal experiments were approved by the Animal Experimental Ethics Committee of Wenzhou Medical University.

\section{Funding}

This work is supported by Zhejiang Provincial Natural Science Foundation of China (No.LY18H160048), Wenzhou Municipal Sci-Tech Bureau Project (No. Y2020158), and National Natural Science Foundation of China (No. 81672385).

\section{Conflict of interest}

The authors declare no conflict of interest.

\section{Publisher's note}

Springer Nature remains neutral with regard to jurisdictional claims in published maps and institutional affiliations.

Supplementary information The online version contains supplementary material available at https://doi.org/10.1038/s41419-021-03456-7.

Received: 28 August 2020 Revised: 14 January 2021 Accepted: 21 January 2021

Published online: 10 February 2021

\section{References}

1. Ghosh, S. Cisplatin: the first metal based anticancer drug. Bioorg. Chem. 88, 102925 (2019).

2. Sun, C. Y., Zhang, Q. Y., Zheng, G. J. \& Feng, B. Phytochemicals: current strategy to sensitize cancer cells to cisplatin. Biomed. Pharmacother. 110, 518-527 (2019).

3. Cho, H. R. et al. Assessment of bevacizumab resistance increased by expression of BCAT1 in IDH1 wild-type glioblastoma: application of DSC perfusion MR imaging. Oncotarget 7, 69606-69615 (2016).

4. Zhang, L. \& Han, J. Branched-chain amino acid transaminase 1 (BCAT1) promotes the growth of breast cancer cells through improving mTOR-mediated mitochondrial biogenesis and function. Biochem Biophys. Res. Commun. 486, 224-231 (2017).

5. $\mathrm{Xu}, \mathrm{Y}$. et al. Overexpression of BCAT1 is a prognostic marker in gastric cancer. Hum. Pathol. 75, 41-46 (2018).

6. Zeng, B. et al. The role of DNMT1/hsa-miR-124-3p/BCAT1 pathway in regulating growth and invasion of esophageal squamous cell carcinoma. BMC Cancer 19, 609 (2019).

7. Lin, X., Tan, S., Fu, L. \& Dong, Q. BCAT1 overexpression promotes proliferation, invasion, and Wnt signaling in non-small cell lung cancers. Onco. Targets Ther. 13, 3583-3594 (2020).
8. Zhu, Z. et al. Tumour-reprogrammed stromal BCAT1 fuels branched-chain ketoacid dependency in stromal-rich PDAC tumours. Nat. Metab. 2, 775-792 (2020).

9. Zhu, W., Shao, Y. \& Peng, Y. MicroRNA-218 inhibits tumor growth and increases chemosensitivity to CDDP treatment by targeting BCAT1 in prostate cancer. Mol. Carcinog. 56, 1570-1577 (2017).

10. Wu, S.H., Zeng, X.-F., Wang, P., Zhou, Y. \& Lin, W. The Expression and Significance of c-myc and bcat1 in Cervical Cancer. Sichuan Da Xue Xue Bao Yi Xue Ban. 49, 725-730 (2018).

11. Zheng, Y.-H. et al. BCAT1, a key prognostic predictor of hepatocellular carcinoma, promotes cell proliferation and induces chemoresistance to cisplatin. Liver Int. 36, 1836-1847 (2016).

12. $\mathrm{Xu}, \mathrm{M}$. et al. BCAT1 promotes tumor cell migration and invasion in hepatocellular carcinoma. Oncol. Lett. 12, 2648-2656 (2016).

13. Wang, Y. et al. Branched-chain amino acid metabolic reprogramming orchestrates drug resistance to EGFR tyrosine kinase inhibitors. Cell Rep. 28, 512-525 (2019).

14. Papathanassiu, A. E. et al. BCAT1 controls metabolic reprogramming in activated human macrophages and is associated with inflammatory diseases. Nat. Commun. 8, 16040 (2017).

15. Wang, Y. \& Zhang, H. Regulation of autophagy by mTOR signaling pathway. Adv. Exp. Med Biol. 1206, 67-83 (2019).

16. Mansfeld, J. et al. Branched-chain amino acid catabolism is a conserved regulator of physiological ageing. Nat. Commun. 6, 10043 (2015).

17. Kim, Y. C. \& Guan, K-L. mTOR: a pharmacologic target for autophagy regulation. J. Clin. Invest. 125, 25-32 (2015).

18. Janku, F., McConkey, D. J., Hong, D. S. \& Kurzrock, R. Autophagy as a target for anticancer therapy. Nat. Rev. Clin. Oncol. 8, 528-539 (2011).

19. Parkhitko, A. A., Favorova, O. O. \& Henske, E. P. Autophagy: mechanisms, regulation, and its role in tumorigenesis. Biochemistry 78, 355-367 (2013).

20. Liu, J., Fan, L., Wang, H. \& Sun, G. Autophagy, a double-edged sword in antiangiogenesis therapy. Med. Oncol. 33, 10 (2016).

21. Chen, S. et al. Autophagy is a therapeutic target in anticancer drug resistance. Biochim Biophys. Acta 1806, 220-229 (2010).

22. Sui, $X$. et al. Autophagy and chemotherapy resistance: a promising therapeutic target for cancer treatment. Cell Death Dis. 4, e838 (2013).

23. Pennati, M. et al. miR-205 impairs the autophagic flux and enhances cisplatin cytotoxicity in castration-resistant prostate cancer cells. Biochem. Pharm. 87, 579-597 (2014).

24. $\mathrm{Yu}, \mathrm{L}$. et al. Induction of autophagy counteracts the anticancer effect of cisplatin in human esophageal cancer cells with acquired drug resistance. Cancer Lett. 355, 34-45 (2014)

25. Zhao, J., Nie, Y., Wang, H. \& Lin, Y. MiR-181a suppresses autophagy and

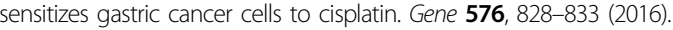

26. Hoffmann, R. et al. Sulfated glycolipid PG545 induces endoplasmic reticulum stress and augments autophagic flux by enhancing anticancer chemotherapy efficacy in endometrial cancer. Biochem. Pharm. 178, 114003 (2020).

27. Tönjes, M. et al. BCAT1 promotes cell proliferation through amino acid catabolism in gliomas carrying wild-type IDH1. Nat. Med. 19, 901-908 (2013).

28. Fang, Y., Vilella-Bach, M., Bachmann, R., Flanigan, A. \& Chen, J. Phosphatidic acidmediated mitogenic activation of mTOR signaling. Science 294, 1942-1945 (2001).

29. Sarkar, S., Ravikumar, B., Floto, R. A. \& Rubinsztein, D. C. Rapamycin and mTORindependent autophagy inducers ameliorate toxicity of polyglutamine-expanded huntingtin and related proteinopathies. Cell Death Differ. 16, 46-56 (2009).

30. Ananieva, E. A. \& Wilkinson, A. C. Branched-chain amino acid metabolism in cancer. Curr. Opin. Clin. Nutr. Metab. Care 21, 64-70 (2018).

31. Sivanand, S. \& Vander Heiden, M. G. Emerging roles for branched-chain amino acid metabolism in cancer. Cancer Cell 37, 147-156 (2020).

32. Ananieva, E. A., Powell, J. D. \& Hutson, S. M. Leucine metabolism in T cell activation: mTOR signaling and beyond. Adv. Nutr. 7, 798S-805S (2016).

33. Nojiri, S., Fujiwara, K., Shinkai, N., lio, E. \& Joh, T. Effects of branched-chain amino acid supplementation after radiofrequency ablation for hepatocellular carcinoma: a randomized trial. Nutrition 33, 20-27 (2017).

34. Shiozawa, S. et al. Impact of branched-chain amino acid-enriched nutrient on liver cirrhosis with hepatocellular carcinoma undergoing transcatheter arterial chemoembolization in Barcelona clinic liver cancer stage b: a prospective study. J. Nippon. Med. Sch. 83, 248-256 (2016).

35. Wolfson, R. L. et al. Sestrin2 is a leucine sensor for the mTORC1 pathway. Science 351, 43-48 (2016).

36. Day, C. P., Merlino, G., Van \& Dyke, T. Preclinical mouse cancer models: a maze of opportunities and challenges. Cell 163, 39-53 (2015). 